\title{
An assessment of interfractional bladder, rectum and vagina motion in postoperative cervical cancer based on daily cone-beam computed tomography
}

\author{
XIN WANG, MIN YU, JIN WANG, RENMING ZHONG, YALI SHEN, \\ YAQIN ZHAO, ZHIPING LI, SEN BAI and FENG XU \\ Department of Radiation Oncology, Cancer Center, West China Hospital, \\ Sichuan University, Chengdu, Sichuan 610041, P.R. China
}

Received November 13, 2014; Accepted September 8, 2015

DOI: $10.3892 / \mathrm{mco} .2015 .704$

\begin{abstract}
Interfractional variations of the bladder, rectum and vagina may affect the accuracy of postoperative intensity-modulated radiotherapy in patients with cervical cancer. This study aimed to assess the interfractional variations with daily $\mathrm{kV}$ cone-beam computed tomography (CBCT). All the patients were instructed to control the filling status of the bladder and rectum. CBCT images were obtained daily after set-up and the bladder, rectum and vagina were contoured on each CBCT scan. All the contours were transferred to the planning $\mathrm{CT}$ following image fusion. Interfractional variations in pelvic organs were assessed with CBCT based on two reference lines, which were identified as A (the midsaggital line across the superior border of pubic symphysis) and B (a parallel line $1.5 \mathrm{~cm}$ above line A). The mean volume (range) of the bladder and rectum was 156.5 (1.7-626.5) and 48.2 (11.3-139.7) $\mathrm{ml}$, respectively. The uniform planning target volume (PTV) margin of $10 \mathrm{~mm}$ failed to encompass the vagina in 17.3 and $18.1 \%$ of the fractions on lines $\mathrm{A}$ and $\mathrm{B}$, respectively. The motion of the vagina (standard deviation) was $0.3(0.3)$ and $0.1(0.5) \mathrm{cm}$ on lines A and B, respectively. The anteroposterior dimension and position of the vagina were significantly affected by the filling status of the bladder $(\mathrm{P}<0.05)$, but not by that of the rectum. Although instructions were given, the interfractional variations of the vagina and other pelvic organs were significant, which
\end{abstract}

Correspondence to: Dr Feng Xu, Department of Radiation Oncology, Cancer Center, West China Hospital, Sichuan University, 37 Wainan Guoxue Lane, Chengdu, Sichuan 610041, PR. China E-mail: fengxu2014@sina.com

Abbreviations: CBCT, cone-beam computed tomography; IMRT, intensity-modulated radiotherapy; OARs, organs at risk; IGRT, image-guided radiotherapy; CTV, clinical target volume; PTV, planning target volume

Key words: cone-beam computed tomography, intensity-modulated radiotherapy, organ motion, postoperative cervical cancer, vagina motion may exceed the uniform PTV margin; therefore, more effective methods to decrease these variations should be investigated.

\section{Introduction}

The use of intensity-modulated radiation therapy (IMRT) for postoperative cervical cancer has increased significantly over the last few years, due to its highly conformal dose distribution to the targets and favorable acute and chronic toxicity profile (1-10). However, during this highly precise radiotherapy treatment, the movement of organs at risk (OARs) and targets may be a major issue and present the physicians with a significant challenge.

There are several studies on radical radiotherapy for cervical cancer, which demonstrated that significant movements of the uterus may be observed due to the filling of the bladder and rectum (11-15). With the different daily filling status of OARs and the steep dose gradient, there is a potential risk of a geographical miss; this may result in a reduction of local control in gynecological cancer of $\leq 30 \%$ (16). For postoperative cervical cancer patients, there may be similar detrimental effects; however, the number of related studies is currently limited.

Cone-beam computed tomography (CBCT)-based image-guided radiotherapy (IGRT) is a technology used for reducing positional set-up errors in radiotherapy. In addition, CBCT may achieve 3D volume imaging of organs, with sufficient soft-tissue contrast to differentiate between these organs. Furthermore, the daily bladder, rectum and vagina motion may be studied based on CBCT scans.

The aim of this study was to determine the interfractional motion and deformation of the bladder, rectum and vagina in patients with postoperative cervical cancer treated with IMRT.

\section{Patients and methods}

Patients. Between January, 2008 and June, 2009, post-hysterectomy patients with cervical cancer requiring postoperative radiotherapy or chemoradiotherapy due to positive pelvic lymph nodes or other high-risk factors evaluated retrospectively. Patients were considered as ineligible if they 
exhibited a positive vaginal cuff, disease outside the pelvis, or mental status alterations. A total of 8 patients receiving IMRT and IGRT in our institution were included in the analysis. The postoperative stages were classified as $\mathrm{Ib}-\mathrm{IIb}$ and the patient age ranged between 42 and 66 years.

Planning CT scan. The planning CT scan was performed in the supine position. A MedTech body frame (Elekta AB, Stockholm, Sweden) and thermoplastic body mask (Klarity Medical, Guangzhou, China) were utilized. The slice thickness was $3 \mathrm{~mm}$ and the scanned volume extended from the anus to L1. All the patients were instructed to control the filling status of the bladder and rectum in order to minimize organ deformation during the treatment. One hour prior to scanning, the patients were instructed to ingest $500 \mathrm{ml}$ of water with the intention of achieving a moderately full and comfortable bladder for contrast CT scan acquisition and during each treatment. The patients were also instructed to encourage bowel movement so as to have an empty rectum prior to the CT scan and each treatment.

Treatment. Following simulation, the CT images were transferred to the Elekta PrecisePLAN (Release 2.10) workstation to design the external beam IMRT plan. The clinical target volume (CTV) delineation was referred to the consensus guidelines of the Radiation Therapy Oncology Group (11) and included the common, external, internal iliac and presacral lymph node regions, the upper $3 \mathrm{~cm}$ of the vagina and the paravaginal soft tissues. The CTV-to-planning target volume (PTV) margin was $1 \mathrm{~cm}$ in all directions. The prescribed radiation dose was $50 \mathrm{~Gy}$, delivered in 25 daily fractions of 2 Gy. All the patients were treated with $6 \mathrm{MV}$ X-ray delivered by the Elekta Synergy S system (Elekta AB).

Prior to each treatment, the patients underwent CBCT scans to obtain daily images and set-up errors. The parameters of CBCT imaging were $120 \mathrm{kV}, 25 \mathrm{~mA}$, with rotation starting from $182^{\circ}$ to end at $180^{\circ}$ for 620 frames. Subsequently, the $\mathrm{CBCT}$ images were matched to the planning CT using the bony alignment of the entire pelvis to obtain set-up errors; if the translational error exceeded $2 \mathrm{~mm}$, the patients' position was corrected by adjusting the treatment couch. Rotation errors were not corrected due to the limitations of the treatment couch.

Definition of the bladder, rectum and vagina on CBCT scans. Following treatment, the CBCT scans and planning CT images were transferred to the TomoCom 3.0 workstation (Elekta AB). The bladder, rectum and vagina during each treatment were contoured on these CBCT scans after the soft tissue value was adjusted to fit for anatomical identification (Fig. 1). The part of the rectum lying between $2 \mathrm{~cm}$ below and $3.5 \mathrm{~cm}$ above the superior border of the pubic symphysis was contoured. The vagina was differentiated by identifying the tissue between the posterior border of the bladder and anterior border of the rectum on daily CBCT scans. Two physicians jointly defined the contours when these organs were not clearly identified on CBCT scans. The CBCT scans were then fused with the planning CT images. The quality of the contours and each registration were reviewed and verified by a senior physician and the structures contoured on the CBCT scans were transferred to the planning CT images.
Analysis of organ motion. The bladder motion was first defined as the movement of the bladder border relative to that on the planning CT. On the midsagittal plane, the vertical distance of the anterior, posterior, superior and inferior bladder borders between the planning CT and each CBCT scan was measured. The bladder motion of the left and right borders was measured on the plane vertical to the posterior side of the pubic symphysis.

In order to assess the motion of the vagina, the posterior bladder border and the anterior rectal border, two reference lines were identified on the axial images using the sagittal and coronal images on each planning CT image. Line A was defined as the midsagital line across the superior border of the pubic symphysis; and line B was the parallel line $1.5 \mathrm{~cm}$ above line A (Fig. 2). The interfractional motion of the posterior bladder border and anterior rectal border were measured on lines A and B, respectively. As the apical extent of the vagina could not be consistently identified on the CBCT scan without implanted markers and the cephalad extent of the vagina was variable according to the differences in the bladder filling status (12), the motion of the vagina in the anteroposterior (AP) direction and the AP dimension of the vagina were measured on lines $\mathrm{A}$ and $\mathrm{B}$, in the midline on which the vagina was more readily identified. The bladder and rectal volumes were also calculated and the correlation between organ motion and changes in these volumes was analyzed.

As regards organ motion, the mean \pm standard deviation (SD) of all the data was calculated. Motion in the posterior direction was defined as positive and in the anterior direction as negative. Similarly, motion in the superior direction was defined as positive and in the inferior direction as negative. The Pearson's correlation coefficient was utilized to investigate the correlation between the margins and the changes in the volumes of the rectum and bladder.

\section{Results}

CBCT images. CBCT ensures adequate image quality to define the structure and boundaries of soft tissue organs, including the bladder, rectum and vagina in the pelvis. A total of 200 sessions of CBCT were performed for the 8 patients, and the structures and boundaries of the bladder, rectum and vagina were delineated on $176 \mathrm{CBCT}$ images that were of good quality. On average, $22 \mathrm{CBCT}$ images/patient were usable for analysis.

Variability of bladder and rectum volume. The repeated CBCT scans revealed extensive organ motion and variations in the volume and shape of the bladder and rectum. The mean volume (range) of the bladder was 158.5 (15.4-626.5) ml, with an SD of $134.3 \mathrm{ml}$. Significant differences were observed between the planning CT scan and the following daily CBCT scans, ranging from -471.0 to $264.4 \mathrm{ml}$. The mean volume on weeks $1,2,3,4$ and 5 was 156.2, 162.5, 143.2, 138.7 and $159.2 \mathrm{ml}$, respectively. However, there were no significant differences among weekly scans due to the instructions $(\mathrm{P}>0.05)$.

The mean volume (range) of the rectum was 48.2 (11.3-139.7) $\mathrm{ml}$, with an SD of $18.9 \mathrm{ml}$. Differences ranging from -71.1 to $102.4 \mathrm{ml}$ compared with the planning CT were observed. The mean volume of the rectum on 

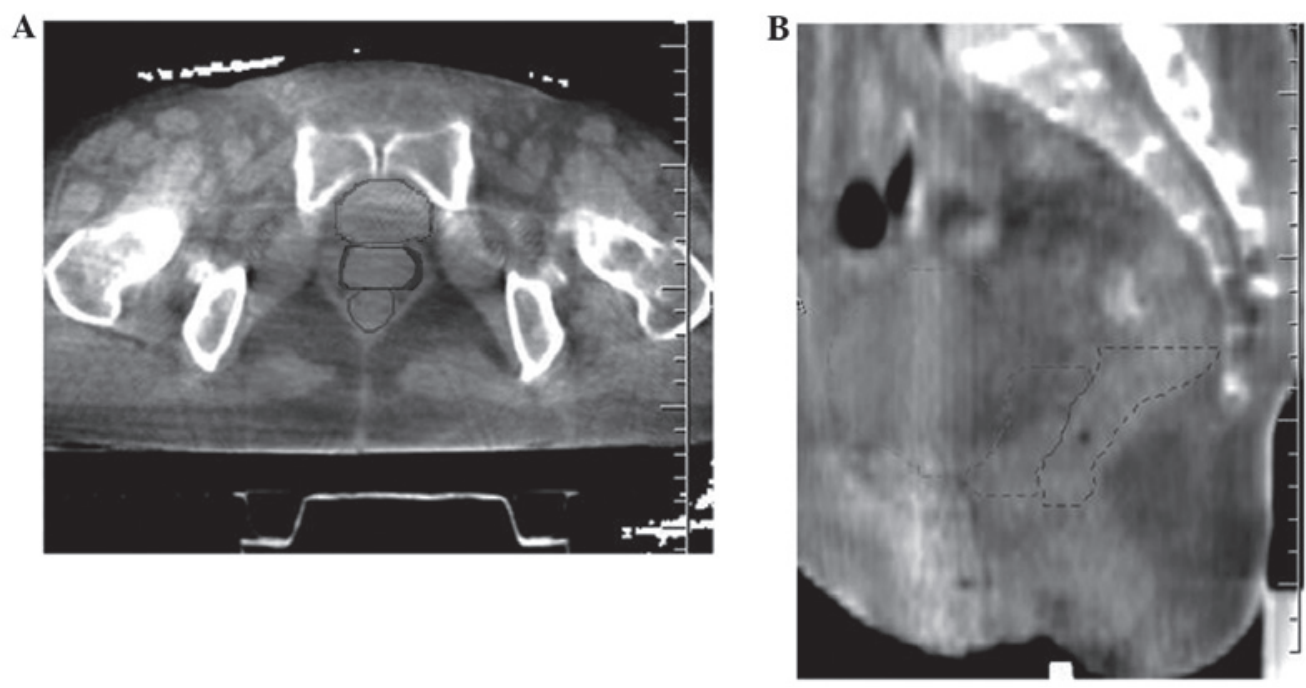

Figure 1. Cone-beam computed tomography image of a patient obtained after conventional set-up presenting the contours of the bladder (anterior), rectum (posterior) and vagina (middle). (A) Transverse plane, (B) midsagittal plane.
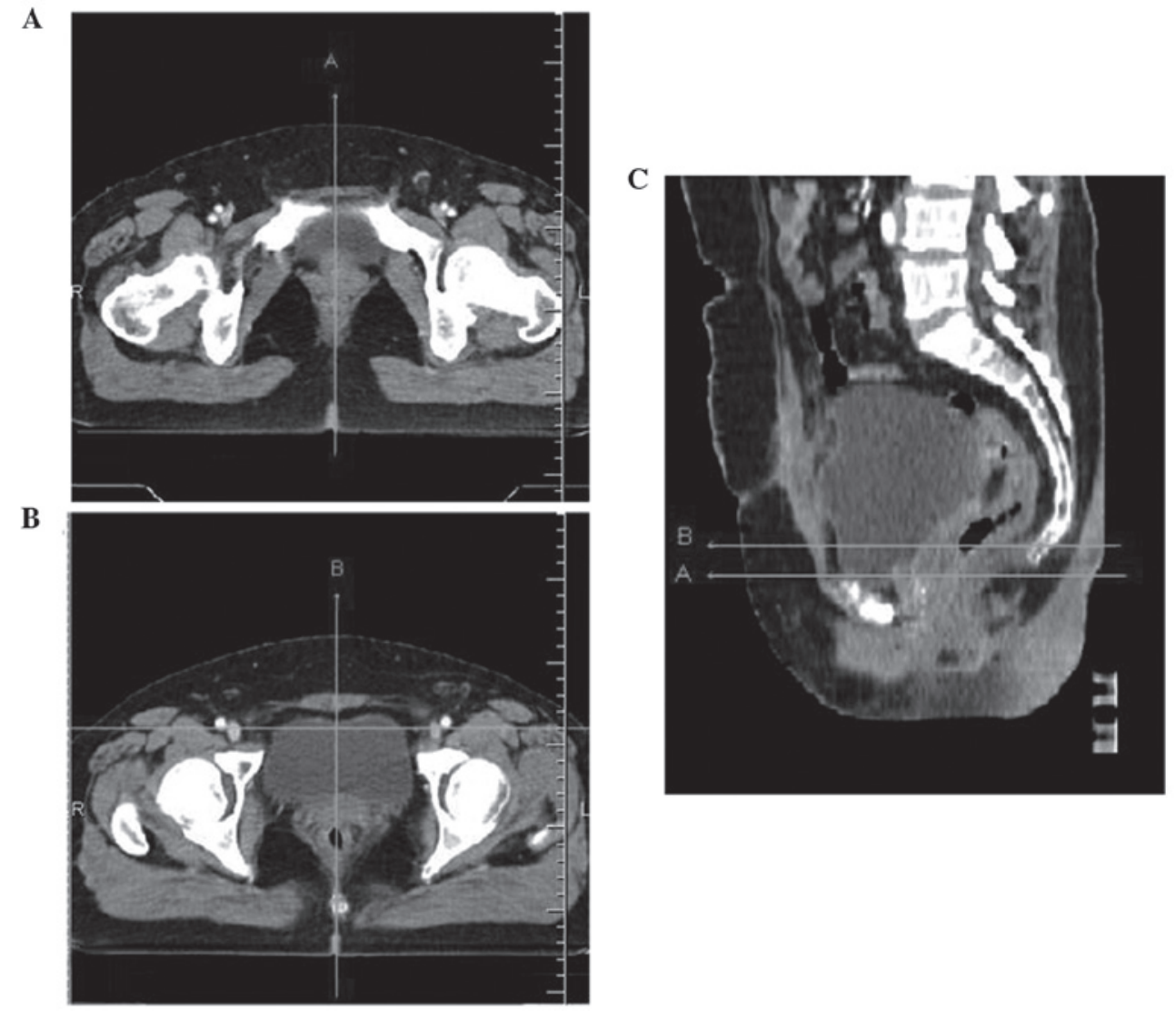

Figure 2. Reference lines delineated to measure the motion of organs and targets. (A) Axial plane across line A (midsagital line across the superior border of the pubic symphysis). (B) Axial plane across line B (parallel line $1.5 \mathrm{~cm}$ above line A). (C) Midsagittal plane of the TomoCom workstation.

weeks $1,2,3,4$ and 5 was $43.5,44.7,50.8,48.8$ and $53.2 \mathrm{ml}$, respectively. The differences among weekly scans were also not significant due to the instructions $(\mathrm{P}>0.05)$.

Motion of the bladder and rectum boundaries. The motion and deformation of the bladder were significant according to the daily CBCT scans. The results of bladder motion are presented in Table I. The motion data of the posterior and superior boundaries, which indicated the most significant movement, are displayed in Fig. 3. The motion of the inferior boundary was found to be the smallest $(0.1 \mathrm{~cm} ; \mathrm{SD}=0.7 \mathrm{~cm})$.

The motion of the posterior boundary was intensively analyzed, as it may significantly affect the delineation of CTV. In addition, obvious motion and deformation of the rectum 
Table I. Bladder motion (cm).

Values Anterior Posterior Inferior Superior Left Right

\begin{tabular}{lrrrrrr}
\hline Mean & 0.5 & -0.7 & 0.1 & -0.9 & -0.5 & -0.5 \\
SD & 0.9 & 1.0 & 0.7 & 3.3 & 0.8 & 0.7 \\
Max & 2.9 & 1.6 & 2.0 & 8.1 & 1.7 & 1.1 \\
Min & -1.6 & -7.2 & -1.1 & -9.1 & -3.6 & -3.2
\end{tabular}

Max, maximum; min, minimum; SD, standard deviation.

A

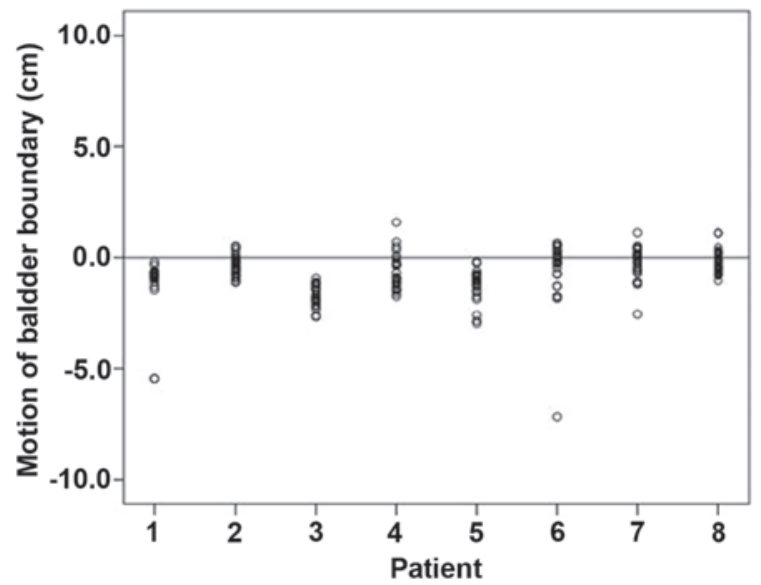

B

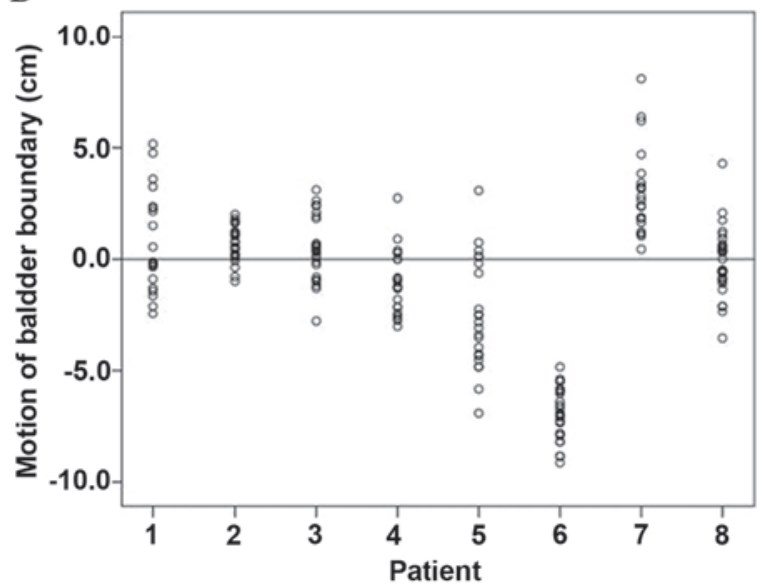

Figure 3. Motion of the (A) posterior and (B) superior bladder boundary compared with the planning computed tomography.

were also observed based on daily CBCT scans. Similar to the posterior boundary of the bladder, only the motion of the anterior boundary of the rectum was intensively analyzed. These two types of motion along lines A and B, respectively, are shown in Table II.

Position of the vagina relative to the PTV. We reviewed the position of the vagina relative to the margins of the PTV on lines A and B based on daily CBCT scans, to assess whether the vagina was adequately covered by the planning. For the 176 sessions of CBCT, the uniform margin of $10 \mathrm{~mm}$ failed to encompass the vagina in 17.3 and $18.1 \%$ of fractions on lines $\mathrm{A}$
Table II. Motion of bladder and rectum boundaries $(\mathrm{cm})$.

\begin{tabular}{lllllr}
\hline & \multicolumn{2}{c}{$\begin{array}{c}\text { Motion of posterior } \\
\text { boundary of bladder }\end{array}$} & & & \multicolumn{2}{c}{$\begin{array}{c}\text { Motion of anterior } \\
\text { boundary of rectum }\end{array}$} \\
Values & Line A & Line B & & Line A & Line B \\
\hline Mean & 0.08 & -0.4 & & -0.5 & -0.7 \\
SD & 1.1 & 0.7 & & 1.1 & 1.2 \\
Max & 2.9 & 1.9 & & 2.1 & 1.7 \\
Min & -1.7 & -2.1 & & -3.9 & -3.7
\end{tabular}

Max, maximum; min, minimum; SD, standard deviation.
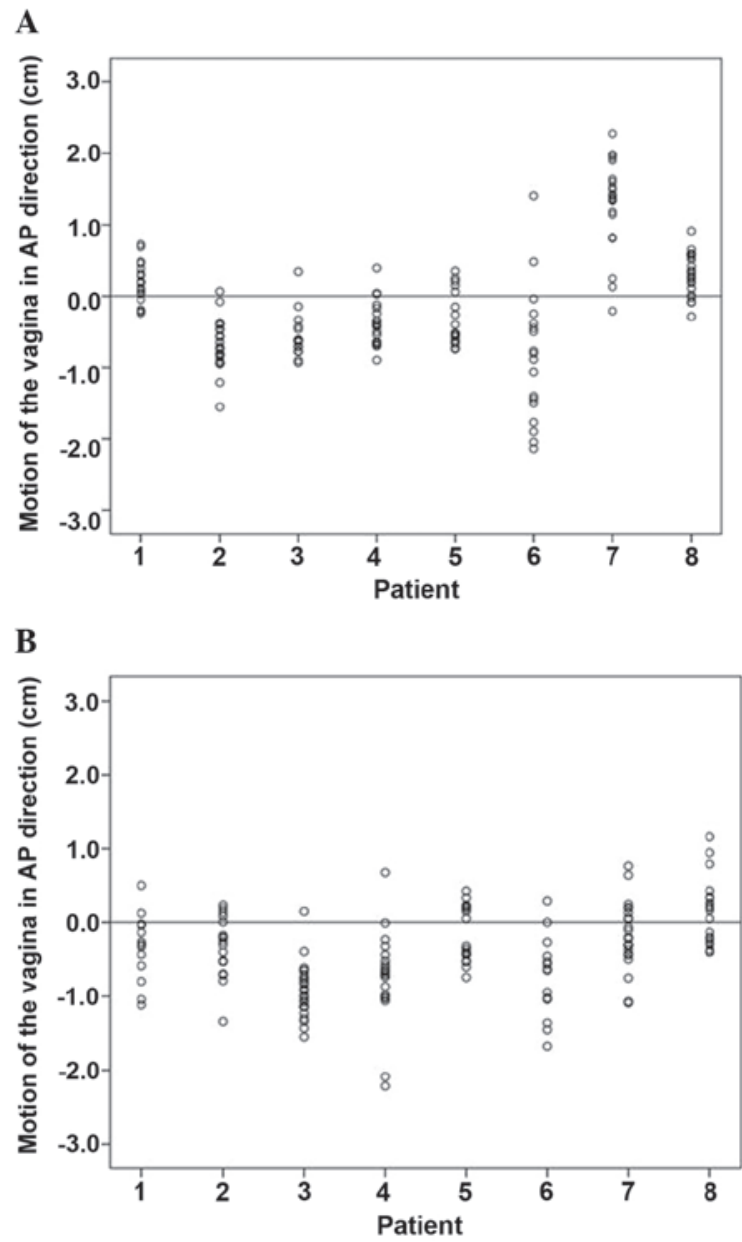

Figure 4. Motion of the vagina in the anteroposterior (AP) direction in the midline along (A) line A and (B) line B. The positive values indicate that the vagina moved posteriorly and the negative values indicate motion anteriorly.

and $\mathrm{B}$, respectively. The variability of the AP dimension of the vagina was used as a representative measure for vaginal deformation and was also measured based on daily CBCT scans. The mean \pm SD of the AP dimension of the vagina was $1.3 \pm 0.8 \mathrm{~cm}$ on line $\mathrm{A}$ and $1.5 \pm 0.9 \mathrm{~cm}$ on line $\mathrm{B}$. The mean (SD) motion of the vagina was $0.3(0.3) \mathrm{cm}$ and $0.1(0.5) \mathrm{cm}$ on lines A and B, respectively; and in the AP direction in the midline the range of motion was -2.1 to $2.3 \mathrm{~cm}$ and -2.2 to $2.1 \mathrm{~cm}$, respectively (Fig. 4). 
Correlation between organ volume and organ motion. The change in the posterior bladder boundary relative to the bladder volume was found to be significant on lines A and B $(\mathrm{P}<0.05$ for both; the Pearson's correlation coefficient was 0.32 and 0.31 , respectively). In addition, the change in the anterior rectal boundary was found to be correlated with the volume of the rectum on lines A and $\mathrm{B}(\mathrm{P}<0.05$ for both; the Pearson's correlation coefficient was 0.29 and 0.18 , respectively).

The change in the AP dimension of the vagina relative to the bladder volume was found to be subtle but significant on lines A and B ( $\mathrm{P}<0.05$ for both; the Pearson's correlation coefficient was 0.37 and 0.30 , respectively). No correlation was found between the change in the AP dimension of the vagina and rectum volume on lines $\mathrm{A}$ and $\mathrm{B}$ ( $\mathrm{P}>0.05$ for both; the Pearson's correlation coefficient was 0.092 for both). The correlation of the motion of the vagina in the AP direction in the midline with bladder volume was found to be weak but significant on lines A and $\mathrm{B}(\mathrm{P}<0.05$ for both; the Pearson's correlation coefficient was 0.37 and 0.44 , respectively). However, no correlation was found between the motion of the vagina and rectal volume on lines A and B ( $\mathrm{P}>0.05$ for both; the Pearson's correlation coefficient was 0.026 and 0.011 , respectively).

\section{Discussion}

The motion and deformation of the target and OARs may significantly affect the accuracy of radiotherapy, particularly IMRT. Quantification of organ motion may help define appropriate PTV margins, optimize target volume coverage of the vagina and spare normal tissues. Previous studies have addressed these problems in patients with advanced cervical cancer (13-16). However, studies on patients with cervical cancer postoperatively are sparse. As regards the methodology for studying motion and deformation, different modalities have been reported, including ultrasound (17), magnetic resonance imaging (MRI) scans $(18,19)$, repeated CT scans and on-board imaging (OBI) (15). CBCT is increasingly used for online set-up correction, soft tissue targeting, safety margin calculation $(20,21)$ and image-guided adaptive radiotherapy $(22,23)$. $\mathrm{CBCT}$ is more convenient for daily imaging compared with MRI and CT. Therefore, in this study, CBCT was used daily to assess the motion and deformation of the bladder, rectum and vagina during postoperative radiotherapy in cervical cancer patients.

In previous studies, large variations and a time trend of bladder filling were reported in patients undergoing radical radiotherapy for cervical cancer when no bladder control instructions were provided (14,24). Ahmad et al (17) reported that the bladder volume ranged from 30 to $770 \mathrm{ml}$. Without instructions, the bladder volume was reported to gradually decrease weekly, and it was usually significantly different by week $4(14,24,25)$. The bladder volume reduction was considered to be a treatment-related side effect (17). For postoperative radiotherapy, the variation of the bladder was also significant. It was reported that, despite instructions regarding the significance and method of pretreatment fluid intake, differences between the pretreatment full bladder and each subsequent CT scan ranged from 53.2 to $698.0 \mathrm{cc}$ (12). In our study, similar results were reported and the differences ranged from -471.0 to $264.4 \mathrm{ml}$. No obvious time trend was observed in our study due to the specific drinking instructions. In order to achieve a more constant bladder volume with instructions, certain researchers suggested a personal drinking advice each treatment day based on the biofeedback (26), or to maintain a smaller initial bladder volume and use a patient information sheet (27).

As regards the rectal volume, the results may be quite different, as different regions of the rectum were delineated in different studies. For patients receiving postoperative radiotherapy, marked variations in rectal volume were observed without instructions, ranging from 46 to $193 \mathrm{cc}$ (12). The average rectal volume reported in our study was smaller, which may be explained by the fact that only part of the rectum was delineated in our study, as the upper and lower parts of the rectum were difficult to differentiate on CBCT scans. However, the difference in volume was similarly significant (range, -71.1 to $102.4 \mathrm{ml}$ ), even with instructions. Another study also reported variations in bowel volume, despite using laxatives to minimize bowel content (28).

It was reported that the movements of the uterus were found to be larger compared with those of the cervix, particularly in the superoinferior (SI) and AP directions (29). Buchali et al (16) revealed that the uterus and cervix moved for $\leq 1.5$ and $0.6 \mathrm{~cm}$, respectively, comparing a full bladder with an empty bladder. Ahmad et al (17) reported that the displacement of the uterus was $2 \mathrm{~cm} / 100 \mathrm{ml}$ bladder volume change. The daily cervical movement represented by 2 seeds placed in the cervix was reported to be 1.9, 4.1 and $4.2 \mathrm{~mm}$ in the right-to-left (RL), SI and AP directions, respectively, using OBI (15). However, these results were all based on patients not treated surgically.

To the best of our knowledge, the number of available studies focusing on vaginal motion in postoperative patients is limited $(12,25,30,31)$. The motion of the vaginal apex in postoperative patients appeared to be more significant compared with the motion of the cervix in patients receiving radical radiotherapy. The motion of fiducial markers placed at the vaginal apex, which represented the motion of the apex, was previously assessed. Harris et al (32) reported that the median motion of the fiducials was $5.8 \mathrm{~mm}$ (range, 0.6-20.2 $\mathrm{mm}$ ) and the directional margins along the RL, SI and AP axes were $3.1,9.5$ and $12.1 \mathrm{~mm}$, respectively, using daily megavoltage CT scans. Jhingran et al (12) reported that the median movement of the markers was $0.59 \mathrm{~cm}$ (range, $0-0.9 \mathrm{~cm}), 1.46 \mathrm{~cm}$ (range, 0.8-2.79 cm) and $1.2 \mathrm{~cm}$ (range, 0.6-2.1 cm) in the RL, $\mathrm{AP}$ and SI directions, respectively, using repeat CT scans.

In addition to the motion of the vaginal apex, the displacement of the vagina may also vary due to the differences in the filling status of the bladder and rectum (12). However, these displacements have not been extensively investigated. It was reported that the vaginal CTVs change their position with a maximum displacement in the AP direction using weekly MRI scans, with a $95 \%$ confidence level of $2.3 \mathrm{~cm}$ (25). In our study, we obtained similar results on vagina motion compared with those in the literature, by measuring the motion on two levels where the vagina could be easily differentiated. In the majority of the cases, the vagina could be differentiated from the bladder and rectum on CBCT scans, with daily $\mathrm{CBCT}$ scans providing accurate daily details on interfractional location and the status of the soft tissues at the treatment time. The motion of the vaginal apex was not assessed in our study, as the apical extent of the vagina could not be consistently 
identified on the CBCT scans, and no marker was implanted to help differentiate the vaginal apex.

To standardize the CTV delineation of the vagina, it was suggested to maintain a $1.5-\mathrm{cm}$ distance between the anterior and posterior borders of the CTV at the midline (11). In our study, we measured the daily AP dimensions of the vagina on two levels in the midline and our results revealed that the mean AP distance of the vagina on lines A and B was 1.3 and $1.5 \mathrm{~cm}$, respectively, consistent with the recommendations.

As obvious movement of the vagina, bladder and rectum was demonstrated, the margin of the target should be carefully considered to avoid the underdosage of the target. In this study, we found that a uniform margin of $10 \mathrm{~mm}$ failed to encompass the vagina in 17.3 and $18.1 \%$ of the fractions on lines $\mathrm{A}$ and $\mathrm{B}$, respectively. However, this encompassment rate appeared to be higher compared with that of intact cervical cancer (33) and the vaginal apex postoperatively (31). For intact cervical cancer, a uniform margin of $10 \mathrm{~mm}$ reportedly failed to encompass the CTV in $59 \%$ of the fractions and failed to encompass the cervix and fundus in 36 and 54\% of the fractions, respectively (33). It was recently reported that the probability of cuff excursion outside the CTV was reduced to $4.2 \%$ as margin size increased to $2.0 \mathrm{~cm}$ in patients receiving postoperative irradiation (31). It was also revealed that interpatient variation was the predominant component of high variation in margin estimates (33). Therefore, it is crucial to individualize the internal target volume (ITV). To measure individualized ITV, certain studies used fused planning CT scans obtained with a full and empty bladder $(11,12)$. However, from our study as well as others, it is known that the difference between the full and empty bladder would be more obvious during the radiation treatment course compared with baseline (12). In order to reduce the uncertainty of target location, filling the bladder with a fixed volume of saline was attempted; however, this may not practical for all patients or entirely tolerable during radiotherapy (12). Apart from the bladder, the filling status of the rectum may also affect the target location, but has been less extensively investigated in gynecological patients. It was reported that a Fleet enema was given to patients with prostate cancer to decrease the uncertainty of the prostate position caused by rectal filling; however, it was not practical for gynecological patients due to the more severe diarrhea and hemorrhoids caused by the larger irradiation volume. Moreover, certain researchers suggested evaluating the rectal volume prior to simulation and trying to limit the variations in rectal volume by advising patients to attempt and evacuate, or by decreasing the gas in the rectum using a rectal tube (12). Otherwise, a 5-10-mm margin would be suggested to be added to the posterior border of the target (12).

The association between bladder and rectal volume and shifts in the position of uterus, cervix and vaginal apex markers were analyzed by several studies $(12,13,16,25)$. The association between bladder filling and uterine movement was controversial $(13,16)$, while rectal filling was reported to be correlated with the movement of the cervix and upper vagina $(13,25)$. Jhingran et al (12) revealed that rectal and bladder volume were both correlated with significant displacement of the vaginal apex. In our study, it was demonstrated that the position of the vagina was significantly affected by the filling status of the bladder, but not by that of the rectum.
The disadvantage of CBCT is the relatively poor soft tissue contrast, particularly for the distal rectum and vagina. Therefore, we only assessed the volume of the rectum that lies between $2 \mathrm{~cm}$ below and $3.5 \mathrm{~cm}$ above the superior border of the pubic symphysis, and assessed the motion of the vagina on line A (the horizontal line across the superior border of the pubic symphysis) and line B (the parallel line $1.5 \mathrm{~cm}$ above line A), which could be easily identified. However, the predominant advantage of CBCT is that it enables the acquisition of daily images and online corrections.

In conclusion, daily $\mathrm{kv}-\mathrm{CBCT}$ scanning may be used to effectively evaluate the interfractional motion and deformation of the OARs and the target during postoperative radiotherapy of cervical cancer. It was demonstrated that there remain obvious variations in target location and organ volume, even with specific patient instructions. In addition, the variations in the filling status of the bladder may significantly affect the position of the vagina. During treatment, it is crucial to consider that any uncertainty regarding the position of the OARs and the target may affect treatment accuracy. The uniform PTV margin of $10 \mathrm{~mm}$ failed to encompass the vagina in 17.3 and $18.1 \%$ of the fractions on lines $\mathrm{A}$ and $\mathrm{B}$, respectively. Therefore, a more effective approach to decreasing this uncertainty is required.

\section{References}

1. Heron DE, Gerszten K, Selvaraj RN, et al: Conventional 3D conformal versus intensity-modulated radiotherapy for the adjuvant treatment of gynecologic malignancies: A comparative dosimetric study of dose-volume histograms. Gynecol Oncol 91: 39-45, 2003.

2. Mundt AJ, Lujan AE, Rotmensch J, Waggoner SE, Yamada SD, Fleming G and Roeske JC: Intensity-modulated whole pelvic radiotherapy in women with gynecologic malignancies. Int $\mathrm{J}$ Radiat Oncol Biol Phys 52: 1330-1337, 2002.

3. Portelance L, Chao KS, Grigsby PW, Bennet H and Low D: Intensity-modulated radiation therapy (IMRT) reduces small bowel, rectum and bladder doses in patients with cervical cancer receiving pelvic and para-aortic irradiation. Int J Radiat Oncol Biol Phys 51: 261-266, 2001.

4. Roeske JC, Lujan A, Rotmensch J, Waggoner SE, Yamada D and Mundt AJ: Intensity-modulated whole pelvic radiation therapy in patients with gynecologic malignancies. Int J Radiat Oncol Biol Phys 48: 1613-1621, 2000.

5. van de Bunt L, van der Heide UA, Ketelaars M, de Kort GA and Jürgenliemk-Schulz IM: Conventional, conformal and intensity-modulated radiation therapy treatment planning of external beam radiotherapy for cervical cancer: The impact of tumor regression. Int J Radiat Oncol Biol Phys 64: 189-196, 2006.

6. Brixey CJ, Roeske JC, Lujan AE, Yamada SD, Rotmensch J and Mundt AJ: Impact of intensity-modulated radiotherapy on acute hematologic toxicity in women with gynecologic malignancies. Int J Radiat Oncol Biol Phys 54: 1388-1396, 2002.

7. Mundt AJ, Mell LK and Roeske JC: Preliminary analysis of chronic gastrointestinal toxicity in gynecology patients treated with intensity-modulated whole pelvic radiation therapy. Int $\mathrm{J}$ Radiat Oncol Biol Phys 56: 1354-1360, 2003.

8. Beriwal S, Jain SK, Heron DE, Kim H, Gerszten K, Edwards RP and Kelley JL: Clinical outcome with adjuvant treatment of endometrial carcinoma using intensity-modulated radiation therapy. Gynecol Oncol 102: 195-199, 2006.

9. Salama JK, Mundt AJ, Roeske J and Mehta N: Preliminary outcome and toxicity report of extended-field, intensity-modulated radiation therapy for gynecologic malignancies. Int $\mathbf{J}$ Radiat Oncol Biol Phys 65: 1170-1176, 2006.

10. Beriwal S, Gan GN, Heron DE, Selvaraj RN, Kim H, Lalonde R, Kelley JL and Edwards RP: Early clinical outcome with concurrent chemotherapy and extended-field, intensity-modulated radiotherapy for cervical cancer. Int J Radiat Oncol Biol Phys 68: 166-171, 2007. 
11. Small W Jr, Mell LK, Anderson P, et al: Consensus guidelines for delineation of clinical target volume for intensity-modulated pelvic radiotherapy in postoperative treatment of endometrial and cervical cancer. Int J Radiat Oncol Biol Phys 71: 428-434, 2008.

12. Jhingran A, Salehpour M, Sam M, Levy L and Eifel PJ: Vaginal motion and bladder and rectal volumes during pelvic intensity-modulated radiation therapy after hysterectomy. Int J Radiat Oncol Biol Phys 82: 256-262, 2012.

13. Taylor A and Powell ME: An assessment of interfractional uterine and cervical motion: Implications for radiotherapy target volume definition in gynaecological cancer. Radiother Oncol 88 250-257, 2008

14. van de Bunt L, Jürgenliemk-Schulz IM, de Kort GA, Roesink JM, Tersteeg RJ and van der Heide UA: Motion and deformation of the target volumes during IMRT for cervical cancer: What margins do we need? Radiother Oncol 88: 233-240, 2008.

15. Haripotepornkul NH, Nath SK, Scanderbeg D, Saenz C and Yashar CM: Evaluation of intra- and inter-fraction movement of the cervix during intensity modulated radiation therapy. Radiother Oncol 98: 347-351, 2011.

16. Buchali A, Koswig S, Dinges S, Rosenthal P, Salk J, Lackner G, Böhmer D, Schlenger L and Budach V: Impact of the filling status of the bladder and rectum on their integral dose distribution and the movement of the uterus in the treatment planning of gynaecological cancer. Radiother Oncol 52: 29-34, 1999.

17. Ahmad R, Hoogeman MS, Quint S, Mens JW, de Pree I and Heijmen BJ: Inter-fraction bladder filling variations and time trends for cervical cancer patients assessed with a portable 3-dimensional ultrasound bladder scanner. Radiother Oncol 89: 172-179, 2008.

18. Chan P, Dinniwell R, Haider MA, Cho YB, Jaffray D, Lockwood G, Levin W, Manchul L, Fyles A and Milosevic M: Inter- and intrafractional tumor and organ movement in patients with cervical cancer undergoing radiotherapy: A cinematic-MRI point-of-interest study. Int J Radiat Oncol Biol Phys 70 $1507-1515,2008$

19. Kerkhof EM, van der Put RW, Raaymakers BW, van der Heide UA, Jürgenliemk-Schulz IM and Lagendijk JJ: Intrafraction motion in patients with cervical cancer: The benefit of soft tissue registration using MRI. Radiother Oncol 93: 115-121, 2009.

20. Hammoud R, Patel SH, Pradhan D, Kim J, Guan H, Li S and Movsas B: Examining margin reduction and its impact on dose distribution for prostate cancer patients undergoing daily cone-beam computed tomography. Int J Radiat Oncol Biol Phys 71: 265-273, 2008.

21. Showalter TN, Nawaz AO, Xiao Y, Galvin JM and Valicenti RK: A cone beam CT-based study for clinical target definition using pelvic anatomy during postprostatectomy radiotherapy. Int J Radiat Oncol Biol Phys 70: 431-436, 2008.
22. Brock KK, Hawkins M, Eccles C, Moseley JL, Moseley DJ, Jaffray DA and Dawson LA: Improving image-guided target localization through deformable registration. Acta Oncol 47: $1279-1285,2008$

23. Ding GX, Duggan DM, Coffey CW, Deeley M, Hallahan DE, Cmelak A and Malcolm A: A study on adaptive IMRT treatment planning using kV cone-beam CT. Radiother Oncol 85: 116-125, 2007.

24. Kerkhof EM, Raaymakers BW, van der Heide UA, van de Bunt L, Jürgenliemk-Schulz IM and Lagendijk JJ: Online MRI guidance for healthy tissue sparing in patients with cervical cancer: An IMRT planning study. Radiother Oncol 88: 241-249, 2008.

25. Jürgenliemk-Schulz IM, Toet-Bosma MZ, de Kort GA, Schreuder HW, Roesink JM, Tersteeg RJ and van der Heide UA: Internal motion of the vagina after hysterectomy for gynaecological cancer. Radiother Oncol 98: 244-248, 2011.

26. Stam MR, van Lin EN, van der Vight LP, Kaanders JH and Visser AG: Bladder filling variation during radiation treatment of prostate cancer: Can the use of a bladder ultrasound scanner and biofeedback optimize bladder filling? Int J Radiat Oncol Biol Phys 65: 371-377, 2006

27. O'Doherty UM, McNair HA, Norman AR, et al: Variability of bladder filling in patients receiving radical radiotherapy to the prostate. Radiother Oncol 79: 335-340, 2006.

28. Lim K, Kelly V, Stewart J, et al: Pelvic radiotherapy for cancer of the cervix: Is what you plan actually what you deliver? Int J Radiat Oncol Biol Phys 74: 304-312, 2009.

29. Kavanagh BD, Schefter TE, Wu Q, Tong S, Newman F, Arnfield M, Benedict SH, McCourt S and Mohan R: Clinical application of intensity-modulated radiotherapy for locally advanced cervical cancer. Semin Radiat Oncol 12: 260-271, 2002

30. Chen MF, Tseng CJ, Tseng CC, Kuo YC, Yu CY and Chen WC: Clinical outcome in posthysterectomy cervical cancer patients treated with concurrent cisplatin and intensity-modulated pelvic radiotherapy: Comparison with conventional radiotherapy. Int J Radiat Oncol Biol Phys 67: 1438-1444, 2007.

31. Ma DJ, Michaletz-Lorenz M, Goddu SM and Grigsby PW: Magnitude of interfractional vaginal cuff movement: Implications for external irradiation. Int J Radiat Oncol Biol Phys 82: 1439-1444, 2012.

32. Harris EE, Latifi K, Rusthoven C, Javedan K and Forster K: Assessment of organ motion in postoperative endometrial and cervical cancer patients treated with intensity-modulated radiation therapy. Int J Radiat Oncol Biol Phys 81: e645-e650, 2011.

33. Tyagi N, Lewis JH, Yashar CM, Vo D, Jiang SB, Mundt AJ and Mell LK: Daily online cone beam computed tomography to assess interfractional motion in patients with intact cervical cancer. Int J Radiat Oncol Biol Phys 80: 273-280, 2011. 